\title{
Philosophical Issues in Experimental Biology
}

Philosophy of Experimental Biology. Marcel Weber, Cambridge, Cambridge University Press. 2004. Hb. $358+x v i$ pp. \$75.00. ISBN: 0-521-82945-3

Ingo Brigandt

Department of History and Philosophy of Science

University of Pittsburgh

1017 Cathedral of Learning

Pittsburgh, PA 15260

E-mail: inb1@pitt.edu

April 9, 2005 (penultimate draft)

Forthcoming in Biology and Philosphy

Traditionally, studies in the philosophy of biology have been strongly centered on evolutionary biology and systematics. To be sure, during the last four decades the field of molecular biology has been subject to substantial philosophical discussion as well. Still, discussions of molecular biology have focused on questions peculiar to this field (such as the relation between classical and molecular genetics). The first exception to this rule was Ken Schaffner's Discovery and Explanation in Biology and Medicine (1993), a monograph which addressed general philosophy of science questions from the point of view of molecular and experimental biology. A decade has passed since Schaffner's seminal account of discovery and theorizing in molecular biology, but recently another book on this topic has appeared: Marcel Weber's Philosophy of Experimental Biology (2004). I feel that Weber's treatment will move the philosophical discussion well beyond Schaffner's original study. Like Schaffner before him, the particular strength of Weber lies in combining two ideals. First, his philosophical discussion is based on detailed case studies from biochemistry, molecular biology, cellular biology, developmental genetics, and neurophysiology. Second, despite close 
attention to actual science, Weber always keeps questions from the general philosophy of science clearly in view.

Some of Weber's discussions pertain to issues that are peculiar to the philosophy of biology. His two chapters on experimental systems and model organisms call attention to the huge body of historical literature on experimental biology that developed in the last decade. While Weber acknowledges that the history of molecular biology can be studied from a sociological, institutional, and economical point of view, he stresses that epistemological and intellectual considerations are vital in understanding the historical development of science. His chapter on developmental biology criticizes developmental systems theory as regards its view of the role of genes in evolution, while largely agreeing that genes do not have a causal primacy in development and that the notion of genetic information is not particularly significant. To be sure, most of Weber's chapters address topics that clearly bear on the general philosophy of science. The important philosophical topics that are discussed in the context of experimental biology are discovery, confirmation, explanation, conceptual change, and scientific realism. Overall, the discussions in different chapters of Philosophy of Experimental Biology are too a large degree independent of each other. I view this as an advantage: each chapter gives a unified and focused discussion of some issues surrounding the particular topic under consideration. For this reason, my subsequent review of Weber's book can address only some of his topics. I shall focus one some of the chapters which I view as being among the most interesting ones and which bear on general issues from the philosophy of science.

\section{Confirmation, inference, and discovery}

Weber's discussion of confirmation and scientific inference (Chapter 4) is based on a detailed analysis of a case from biochemistry - the oxidative phosphorylation controversy. The question was how the metabolic pathway works in which the cell uses the energy derived 
form respiration. From 1961 onwards, the novel chemiosmotic hypothesis competed with the more established chemical hypothesis, but not until 1977 was the debate settled in favor of the chemiosmotic theory (leading to a Nobel prize for Peter Mitchell). Weber argues that the reason that the dispute could not be settled for more than a decade had nothing to do with issues such as underdetermination and Duhem's problem of auxiliary hypotheses. Instead, both theories had advantages and disadvantages in accounting for the experimental evidence, and there was no agreement on the relative significance of these empirical merits. The debate ended once in vitro systems were developed that did not admit an interpretation of the experimental results in terms of the chemical hypothesis. Weber goes on to discuss how different philosophical models of confirmation and scientific inference handle this case. Weber dismisses Bayesianism as an adequate account, arguing that it would have made problematic normative suggestions about theory acceptance. On plausible assignments of prior probabilities and likelihoods, Bayesianism would in fact have recommended accepting the theory that turned out to be true. However, on the Bayesian approach this theory should have been accepted much earlier (in 1966) — at a point where the total evidence did not favor one hypothesis over the other. Then the merits of Deborah Mayo's (1996) error-statistical theory are analyzed. Weber rightly points out that Mayo's approach fits the practice of experimental biology much better than Bayesianism, as Mayo does not assume that scientific inference solely consist of a confirmation relation between evidence and hypothesis, instead it is important to take into account how evidence is produced in a piecemeal fashion. Mayo assumes that there are different levels of inquiry where different types of hypotheses are tested independently: primary hypotheses, experimental models, and data models (Weber applies these notions to his case). In addition, biological experimentation attempts to control for and eliminate possible errors, which aligns with Mayo's stress on error testing. The oxidative phosphorylation controversy was settled once an experimental system had been developed that could control for a possible error in the chemiosmotic interpretation of the 
results. Still, Weber makes plain that Mayo's statistical notion of error and severe test cannot apply to experimental biology, because it is unclear what the relevant reference class for such an experiment is, so that no error frequencies can be assigned. In experimental biology, controlling for error means to control for problematic causal influences that could lead to wrong interpretations of the experiment. Weber concludes his discussion of confirmation by stating that "Mayo commits the same mistake as the Bayesians in seeking formal unity in scientific inference.” (123). In a similar vein, his subsequent chapter on experimental systems and experimental practice (Chapter 5) rejects the idea that epistemic norms are ahistorical, universal rules.

I agree with Weber that formal accounts such as Bayesianism or statistical testing give a highly incomplete account of inference in the context of experimental biology. But I think that philosophical analysis can go further than that. We need to embed these ideas into a more general philosophical framework on scientific inference, so that we have an account of what scientific inference is if it is not identical to the application of general and formal rules - a question that Weber does not address. John Norton (2003) recently argued for a "material theory of induction." In contrast to formal theories of induction, Norton argues that there are no universal inference schemas. Inductive inferences in science are grounded in matters of fact - the 'material' of the induction — that hold only in particular domains. This account fits with Weber's conclusion about the oxidative phosphorylation debate. In addition, Norton is a philosopher of physics, so that the idea that induction is material rather than formal is not peculiar to experimental biology, but a quite general feature of scientific inference. Whether a particular inference is good or not essentially depends on those facts that are pertinent to the matter of the particular induction, so that formal accounts of scientific inference give an incomplete account by leaving out an important factor that determines the quality of the inference. New scientific knowledge generates new inferential power, but not by yielding new abstract schemas of inference. 
I want to go a little further than Norton by stressing the notion of material inference. While on the traditional picture an inference is good because of its form, a material inference is an inference that is good because of its content. The content of the particular premises and conclusions involved determines the quality of the inference, where this content involves empirical and typically substantial scientific knowledge. ${ }^{1}$ My view is that scientific inference is material inference. More specifically, I assume that the concepts occurring in the inference determine the quality of the inference, as the meaning of scientific terms embodies crucial empirical knowledge. Weber's discussion on inference and conformation rightly hints at the fact that formal schemes such as Bayesianism or statistical testing yield an incomplete philosophical account, and that instead scientific inference is local, i.e., domain-specific. The notion of material inference that I pointed at can explain why scientific inference can be rational without conforming to a particular formal scheme.

The notion of material inference also sheds some light on Weber's discussion of scientific discovery (Chapter 3). Weber starts out by discussing Ken Schaffner's (1974) former position, according to which discovery uses the same logic as confirmation - in fact deductive reasoning. Weber discusses the same case that Schaffner used to support his account, the discovery of genetic regulation in the lac operon by Jacob and Monod. Unsurprisingly, Weber rejects Schaffner's deductive interpretation of this instance of discovery. Instead, the very proposal of the repressor model of the lac operon was based on a sort of analogical reasoning: it had been known that repression exists in some enzyme systems, and Jacob and Monod suggested that repression might occur in this case of gene regulation. Weber points out that this is a form of reasoning that is prohibited in the context of justification, since the fact that one mechanism occurs in one system does not entail that it is likely that it will obtain in another, very different system. Then Weber discusses Lindley Darden's (1991) analysis of the history of Mendelian genetics. Using ideas from artificial intelligence, Darden puts forward a set of general 'strategies' of discovery, which can be used 
in various scientific fields. Darden herself acknowledges that the historical record does not permit to settle the question of whether Mendelian geneticists actually used these strategies, but her idea is that they could have been used to arrive at the new hypotheses. However, Weber rightly argues that the important philosophical question is whether general and domain-unspecific rules of discovery are actually used by scientists, so that it is necessary to show that Darden's or at least some other general rules have been used in this historical case. As a final case study, Weber offers a detailed discussion of the discovery of the urea cycle by the biochemist Hans Krebs. As all of Krebs's lab notebooks have been analyzed by the historian Frederic Holmes, this is one of the most closely studied cases of discovery in experimental biology, and different accounts of some steps of discovery haven been given (Grasshof and May 1995; Holmes 1991, 2000). Weber argues that general strategies or heuristics did not play an important role in devising Krebs's new hypothesis. Possibly a sort of analogical reasoning (as in the lac operon) was involved, but arbitrary analogies are easy to find. Background information that is specific to the particular case and the scientific field must always enter the reasoning process in discovery.

Weber's overall conclusion is that the discovery strategies that are used in experimental biology are not general and domain-unspecific rules. I completely agree with Weber on this issue, and at least for the particular case studies chosen - which I take to be representativeWeber offers detailed evidence that formal schemes and general rules yield an insufficient account of both discovery and confirmation. Moreover, Weber appears to assume that the modes of reasoning used in discovery are not identical to the epistemic principles used in confirmation. Still, what he proposes

is not a return to the older view that the generation of theories is an irrational process that is not open to philosophical analysis, or inaccessible altogether. For to show that a kind of reasoning can be rational ... is not the same as showing that it employs general rules or procedures. (86) 
However, Weber has not offered yet an account of how reasoning in discovery can be rational if it neither consists in the use of general rules nor the employment of the inferences used in confirmation. But consider again the notion of a material inference. If we assume that reasoning in the case of scientific discovery is based on material inferences as well, we have a basic motivation for the assumption that reasoning in discovery is rational. The idea that the quality of any type of inference essentially depends on the content of the concepts involved in the premises and the conclusion means that inference and reasoning is local and contextdependent. It suggests that the various individual inferences used are quite different from each other and cannot be subsumed by a limited set of general rules. The general notion of a material inference does not exempt us from giving a detailed account of how scientific reasoning works in concrete cases and what makes a particular inference justified. But it provides elbow room for the assumption that reasoning processes involved in both confirmation and discovery belong to a genus — rational inference — despite the fact that scientific inferences are context-dependent and the various inferences used in discovery and confirmation differ from each other.

\section{Conceptual change}

In his chapter on reference and conceptual change (Chapter 7), Weber discusses the history of the gene concept. To this end, he makes use of Philip Kitcher's (1978) account of conceptual change, which Kitcher (1982) himself applied to the gene concept (Weber offers a much more detailed discussion of this case). Kitcher points out that there are different ways to refer to a category, i.e., there are different modes of reference. There are causal modes of reference, where reference is fixed along the lines of the causal theory of reference, and there are descriptive modes of reference, where the referent is picked out by a description. Kitcher calls the totality of modes of reference for a term the reference potential of this term, and emphasizes that the reference potential of a theoretical term is heterogeneous in that it consist 
of many modes of reference. His fundamental tenet is that concepts are reference potentials, so that conceptual change is the addition of new modes of reference to the reference potential or the deletion of problematic modes of reference.

Weber starts out his discussion of the gene concept by giving a brief outline of the history from 1860 to 1960 . Then he suggests that six different gene concepts were used at different periods of history: the pangene concept, the unit-character concept, the classical gene concept, the neo-classical concept (cistron), the molecular and the contemporary gene concept. Even though this account makes intuitive sense, Weber does not offer any account of how to individuate concepts or a justification of why these are actually different gene concepts. In fact, he does not give a detailed discussion of these gene concepts and their differences; he rather lists them in a table. One issue is that several of Weber's postulated concepts have the same identity criteria of genes. However, a traditional semantic assumption is that there is nothing more to a concept than the conditions that determine what falls under the concept, so that different genes concepts ought to have different identity criteria. Moreover, even appeal to Kitcher's overall philosophical framework on conceptual change would be of no help in trying to justify why these are distinct concepts. For on Kitcher's account for each term there is simply a reference potential that changes over time, but Kitcher does not intend to break the reference potential down into different concepts.

More detailed analysis is given by Weber's subsequent study as to how the reference potential and the reference of the term 'gene' changed. This discussion offers several philosophical insights. Weber spells out in detail five different descriptive modes of reference, i.e., descriptions that were used to fix the reference of the gene concept at different stages in history from 1900-1960. An important feature is that these different modes of reference have different extensions. For instance, in early Mendelian genetics the extension of 'gene' included anything generating Mendelian patterns of inheritance, including groups of genes or larger chromosomal segments. With the Morgan school the extension contracted as the 
concept included a more refined definition, but the extension expanded again with the origin of bacterial genetics. While Kitcher's (1982) brief discussion of the gene concept already hinted at the fact that the reference of the gene concept changed in the course of history, Weber is much more explicit about this and offers a more detailed account. On Weber's account, the change of the gene concept was characterized by what he calls floating reference. In addition, Weber makes clear that there is not one natural kind, 'the' genes. Instead there are several distinct, though strongly overlapping, natural kinds and "geneticists simply switched freely between different modes of reference and frequently changed the set of things they were referring to when using the term 'gene'." (224). A full section is devoted to a discussion about the relation between classical and molecular genes in Drosophila. Even though most genes belong to both types of genes, Weber discusses examples of genes that fall under the classical gene concept, but not under the molecular gene concept (and vice versa). Moreover, he makes a case for the important point that the classical gene concept is still used in molecular genetics, at least for the experimental purpose of identifying (molecular) genes.

From the point of view of semantics, Weber's discussion leaves a few questions unanswered. He presents five descriptive statements that he takes to be modes of reference. It is quite plausible that these were in fact relatively prevalent modes of reference used at a particular period of history. Still, unlike a merely historical discussion, a philosophical account of conceptual change has to offer an argument of why these statements were modes of reference. As Weber acknowledges, if a statement is reference fixing (a descriptive mode of reference) in a certain context, then it is an analytic statement. If it is not a mode of reference, then it is a synthetic statement - it merely expresses a belief about genes without influencing the reference of the term 'gene' (reference is fixed by prior descriptions or causal modes of reference). This difference between statements that fix reference and other statements (between analytic and synthetic statements) is important for the following reasons. If a description is reference determining, then reference-failure is possible if no entity falls 
under this particular description. If, in contrast, the statement is not reference fixing, then geneticists can still refer to genes while making wrong claims about them or even disagreeing about their properties - it is significant that scientists can make opposing claims while still talking about the same entity. Weber's important philosophical claim that the gene concept referred to a particular class of entities at a certain time and referred differently across time depends on showing that certain descriptions were in fact reference fixing. For instance, Weber claims that the term 'unit-character' (as used routinely before the Morgan school) was non-referential, as a descriptive mode of reference obtained. This is an interesting idea, but one surely has to make plausible why it is not the case that 'unit-character' referred to certain entities that were involved in Mendelian inheritance (e.g., because this term referred in a causal fashion to a natural kind). Surprisingly, before presenting his five modes of reference, Weber states that he

will be concerned not so much with the meaning of the gene concept, but with its reference. Within the semantic framework that I am using here, problems in applying the analytic/synthetic distinction pertain mainly to considerations of meaning. (206)

But Weber cannot have his 'mode of reference' cake and eat it. The account is not simply about reference, it is about conceptual change, i.e., about which descriptions were modes of reference. On Kitcher's (1978) account the notion of a mode of reference is a proxy for the meaning of a term - a concept is the set of modes of reference used. Thus, if conceptual change is change is the acquisition or abandonment of modes of reference, then Weber is committed to showing why a certain description is reference determining, i.e., analytic rather than synthetic. Weber's five modes of reference are intuitively plausible—-but the philosophically significant achievement would be to have an argument for this. ${ }^{2}$

Weber's discussion of the history of the gene concept clearly offers important insights into the way in which the reference of the gene concept changed. Still, I want to point to additional important features that an account of conceptual change could include and which- 
in my view-future studies should address to a larger extent. ${ }^{3}$ An account of conceptual change should be able to track the historical development and the change of concepts - in addition to merely studying change of reference. Weber indicates that he assumes that the meaning of the term 'gene' changed substantially, so that this term corresponded to different concepts at different times (such as the classical, the neo-classical, the molecular and the contemporary gene concept). We saw above that Weber did not offer an account of concept individuation, but the important task is clearly to develop a general account that puts us in a position to say why these are actually different concepts and why it is important to recognize that they are different concepts. An account of conceptual change should identify the point in history at which the term 'gene' split into two concept (the molecular and the classical gene concept still being used), and it would be desirable to explain why conceptual change or a conceptual split occurred.

Moreover, Weber follows Kitcher in identifying concepts with reference potentials. Kitcher (1978) developed his framework in order to deal with the incommensurability problem. For this purpose, an account of reference is important, and consequently, Kitcher defines concepts as reference potentials and views conceptual change as the acquisition and abandonment of modes of reference. However, there is more to scientific concepts than a speaker's ability to refer to a category. For instance, consider natural kind terms. The different members of a natural kind share certain causal capacities or are governed by important theoretical principles. As is well known, this is of fundamental importance as it permits the projectability of properties - for a large range of properties, if some members of a kind have a certain property, then it is likely that another member has this property as well (Boyd 1991). However, semantic discussions of natural kind terms have focused on their reference, in particular on the causal theory of reference. But the projectability of property shows that natural kind concepts support important inductive inferences. Weber emphasizes that geneticists referred to different natural kinds at different times or in different context, and he 
mentions that this floating of reference was practically useful (224). But what is important about the advent of a new gene concept is not the ability to refer to a new natural kind (another type of genes) as such, the crucial thing is that the new concept supports new scientifically significant inferences. Different types of inductive inferences may be relevant in different theoretical contexts, so that it can be fruitful to use different gene concepts (or variants of a gene concept) in different situations. Changing and floating reference is simply a consequence of this shift in meaning. Thus, in addition to viewing concepts exclusively as modes of reference - the ability to refer to a category in a certain way - it is important to recognize that concepts figure in scientific inference and that new or changed concepts improve scientists' ability to infer and justify. The same point applies to scientific explanation. The introduction of a novel concept such as 'natural selection' permits scientists to refer to a new kind or a new process (natural selection in this case). But the most important feature of the introduction of the concept of natural selection is that it enabled scientists to explain phenomena that previously could not have been adequately explained. Likewise in the case of a changed concept: the molecular gene concept (in combination with other concepts) supports a range of explanations in molecular biology that could not have been put forward using the classical gene concept only.

Concepts figure in scientific reasoning such as inference and explanation, and a crucial aspect of conceptual change is that novel or changed concepts facilitate scientific discovery, permit scientists to justify new hypotheses, and to explain new ranges of phenomena. In fact, this is an important aspect of conceptual progress. An approach that defines concepts as reference potentials only focuses on a scientist's ability to refer. But in addition to referring, concepts support inferences and explanations. ${ }^{4}$ In sum, my suggestion is that in order to obtain a complete theory of conceptual change and progress, philosophers should work towards an account of concepts that captures the inferential and explanatory potential of concepts. Kitcher (1982) rightly views his discussion of the gene concept as going beyond 
traditional debates as to whether the classical gene concept can be reduced to molecular genetics. On his account, "molecular genetics refines the concept of the gene" by adding new modes of reference (339). But in addition to this refinement, it would be desirable to have a detailed account of the way in which the molecular gene concept supports explanations that the classical gene concept does not yield. While it is widely agreed that the transition from classical to molecular genetics is an instance of explanatory progress, an account of concepts and conceptual change is needed that can show that this progress is due to the emergence of a novel gene concept.

\section{Reductionism and explanation}

In his discussion on reduction and explanation (Chapter 2), Weber endorses a strong

reductionist position. ${ }^{5}$ His core idea is that experimental biology is heteronomous: explanations in this field essentially depend on the knowledge from other fields, in fact, the laws of other fields, namely, macromolecular physics and chemistry. While traditional models of reduction were models of theory reduction and assumed that a high-level theory could be logically derived from a low-level theory, this is not Weber's line. Instead, his approach is better termed explanatory reduction, and he does not assume that reductive explanations proceed by translating the concepts of experimental biology into concepts of physics and chemistry. This is illustrated by his main example: action potentials in neurotransmission. An account of how this mechanism works has to make recourse to entities above the molecular level: neurons, axons, and synapses. Still, while these concepts from different fields of experimental biology (neuroanatomy, cell biology ...) figure in reductive explanations, Weber's central point is that these concepts are descriptive, but do not have explanatory relevance. Instead, the explanatory force resides in the laws of physics and chemistry. In the particular case of action potentials, these are laws from electrochemistry such as the Nernst equation. Weber's picture is that the concepts from experimental biology give a description of 
the mechanism (the spatial arrangement of cellular components). But these descriptions simply the state the initial and boundary conditions of the explanation, while the laws from physics and chemistry do the relevant explaining: "It is these covering laws that have explanatory force; the rest of the mechanistic model basically states how the physicochemical theory should be applied" (26). Thus, experimental biology is heteronomous in that explanations in this field must involve laws from lower-level disciplines.

At first sight, Weber's account sounds like the deductive-nomological (D-N) model of explanation, as he talks about 'initial and boundary conditions' and 'covering laws.' In fact, he explicitly refers to the D-N model when explaining his account. Still, Weber claims that his "thesis is not tied to a particular account of scientific explanation" (27). While he does not offer a real discussion of why his approach is not committed to the D-N model and what alternative philosophical theory of explanation he prefers, my suggestion is to interpret his approach as a combination of the covering law approach to explanation and mechanistic models of explanation in molecular biology. Weber shares some assumptions with the D-N model by using the idea of initial and boundary conditions as mere descriptions and laws as the explanatorily relevant feature. Still, this is just a covering law approach and Weber is not committed to the more substantive claims of the D-N models, such as the idea that explanations are deductive inferences (and a fortiori arguments) and the explanationprediction symmetry. In addition to this, Weber uses the basic framework of mechanistic approaches to explanations, as he assumes that molecular biologists explain by putting forward mechanistic models of biological processes: “the terms 'neuron' and 'axon' stand for bona fide biological concepts, and their descriptive content plays a role in mechanistic explanations" (27). However, traditional accounts of mechanisms have usually assumed that a mechanism explain simply because the entities in a mechanism are connected by activities (Machamer et al. 2000) or by the presence of causal arrows in mechanism diagrams (Delehanty forthcoming). This is not particularly illuminating, and Weber's account improves 
on traditional mechanistic models of explanations by arguing that laws provide the necessary explanatory import. I agree that the employment of physicochemical laws is an important way in which some accounts in experimental biology gain explanatory relevance and thereby become explanations.

I take issue with Weber's reductionist dictum that "The explanatory force is provided solely by the physicochemical theories" (28). The limited validity and significance of his position becomes clear once one inquires as to whether his account yields type-type or tokentoken reduction. This distinction was introduced in the context of traditional models of theory reduction (Fodor 1975; Kimbrough 1978), but it applies to models of explanatory reduction as well. Token-token reduction is very weak notion of reduction, it is about whether we can explain a token biological phenomenon - e.g., the biological processes of a single organismin a reductive fashion. The stronger case of type-type reduction obtains when it is possible to offer a reductive, though unified explanation that covers many instances of a type biological phenomenon, e.g., whether it is possible to offer an reductive account of a biological process as it occurs across different species. Traditionally, arguments against type-type reduction (in the philosophy of biology as well as the philosophy of mind and social science) have relied on the notion of multiple realizability of higher-level kinds. For instance, crucial concepts from classical genetics such as 'gene' or 'dominance' cannot be meaningfully reduced to the notions of molecular genetics as the phenomenon of dominance can be brought about be very different processes on the molecular level (Hull 1974; Kimbrough 1978; Kitcher 1984). Traditional debates about reduction have focused on the question of type-type reduction, typically on models of theory reduction as a particular approach to type-type reduction (Ruse 1973; Goosens 1978; Schaffner 1993; Frost-Arnold 2004). The reason is that token-token reduction is quite trivial, as it follows from physicalism. According to physicalism, a particular organism consists of nothing but molecules and their interactions. For this reason, given a biological process taking place in an individual organism one can view its higher- 
level modules (tissues, cells) as collections of molecules, and once we give a mechanistic account of how these molecular entities interact, this token process is explained in a reductive fashion (Delehanty forthcoming). Everyone in the reductionism debate agrees that tokentoken reduction is possible, as nowadays no one is willing to deny physicalism by endorsing a sort of vitalism.

Weber is not explicit about the distinction between token-token and type-type reduction. But as it turns out, his reductionist account does not yield more than token-token reduction. This becomes clear in his section on multiple realization. While Weber is aware of the fact that the notion of multiple realization was used as an argument against reduction, he does not think it affects his account:

I claim that this [old multiple realization] argument against reductionism is irrelevant. In order to see this, consider the concept of chemotaxis again. ... the fact that chemotactic behavior is multiply realizable does not affect the reductionistic explanation of this organism's behavioral biology. (48)

Thus, as Weber focuses on reductively explaining the processes going on in individual organisms, his account is actually a version of token-token reduction. One problem with this move is that he addresses a different sense of reduction than the disputants in the traditional debate about reduction. It is not surprising that multiple realization is irrelevant for Weber's account, as multiple realization is an argument against type-type reduction only. It can be adequate to choose to defend a different notion of reduction than former accounts, but there are two more substantial problems for Weber's position. First, token-token reduction follows directly from physicalism and Weber therefore defends a position that is trivial given that there are no vitalists around. Second, Weber views his position as a general account about the nature of explanation in experimental biology. However, there are many important explanations in experimental biology that account for biological mechanisms across different species, so that a reductionist position has to argue for some version of type-type reduction. 
This can be most clearly seen by taking a look at developmental biology. Explaining development - as understood by many developmental biologists - means to have an account of the development (ideally the total development) exhibited by the organisms belonging to a taxon. The goal is to understand the developmental principles that govern groups of organisms, such as vertebrate development. Weber actually claims that his reductionist account holds for developmental biology as well. His later chapter on developmental biology (to be discussed in the next section) gives a brief review of pattern formation in Drosophilaunsurprisingly, developmental genetics in a single model organism. Based on this example Weber concludes: "The other important conclusion to be drawn from this section is that current explanations of ontogenetic processes are fully compatible with the kind of reductionism defended in Chapter 2" (255). No one denies that research on individual model organisms is crucial for technological purposes such as biomedical applications, and that it is an important experimental tool for arriving at explanations of development. But attempting to explain the development of single organisms is only one relevant scientific goal, the much more interesting and ultimate intellectual goal is to explain the development of larger groups of organisms. ${ }^{6}$

As Weber present a nomological-mechanistic model of explanation for token processes, it is not clear whether this model applies to types of processes. This essay is not the place to offer a defense of a non-reductionist position, but let me briefly indicate why a reductionist account is not promising for the explanation of developmental processes across species. ${ }^{7}$ Organisms are structured in a hierarchical and modular fashion: individuals are composed of and develop based on developmental modules, and these modules exist on different levels of organismal organization, where one module can consist of different modules on lower levels (e.g., cells). The reason why different levels of organization are to be recognized is that the same developmental module can be present across various species even though its underlying basis on lower levels varies from species to species. For instance, a module as occurring in 
different species may originate due to substantially different developmental processes and involving different genes. Developmental modules are not just brought about by developmental processes, the important point is that they influence subsequent development. A developmental module has a stable causal influence and determines which further developmental events take place - despite the fact that its particular internal structure and what constitutes its causal properties on lower levels may vary from species to species. For this reason, explanations of the development of groups of organisms have to attach to these modules, as long as they have a stable causal influence across species while the causal features of their components changes from case to case. Whether it is a module from a higher level or rather from a lower level of organization that is explanatorily most relevant varies from case to case - it is an empirical question which modules are causally most important. Weber's notion of the heteronomy of experimental biology refers to the idea that explanations in this field must appeal to the knowledge of other fields. I agree that there is an important sense in which developmental biology is heteronomous, but it is not Weber's 'downwards heteronomy,' according to which the explanatory force comes exclusively from the scientific fields studying the lowest levels of organization (physics and chemistry). Adequate developmental explanations are multi-level explanations in that they have to make recourse to the causal properties of entities on different levels of organization, and therefore have to make use of the knowledge from different fields. Developmental biology is heteronomous in that it is based on multi-disciplinary research.

Weber's central claim is that "explanation that really takes biological systems down to physicochemical laws is the goal of much twentieth- and twenty-first-century biological research" (29). This may be one goal of biology (maybe not a goal in itself), but it is clearly not the only goal. A crucial goal is to have an account of biological processes that covers different species, which is particularly relevant for developmental biology. I indicated why I think that reductionist explanations are not sufficient to meet this goal and why adequate 
developmental explanations have to make reference to higher-level developmental modules and their causal properties. An important aim of scientific explanation is to discover the most salient and relevant causal features, and entities above the molecular level can have a stronger causal influence and be less indispensable than causal connections on the lowest level. While Weber focuses on the application of the laws of physics and chemistry to mechanisms in individual organisms or species, he is not concerned with the causal regularities that govern the development and other biological processes across species. Thus, his reductionist account of explanation fails to cover types of explanations that of crucial concern of many experimental biologists, in particular developmental biologists.

\section{Conclusion}

My critical discussion of Marcel Weber's Philosophy of Experimental Biology could only address some of his chapters. This is a very rich book, dealing with various issues - questions peculiar to experimental biology as well as basic topics from the general philosophy of science. Even though not all of his ideas will be viewed as correct or useful, many of Weber's points and arguments will clearly serve as a point of reference for future philosophical discussions on molecular biology. Philosophy of Experimental Biology ought to be a concern for everyone interested in the philosophy of biology.

\footnotetext{
${ }^{1}$ The notion of material inference has been stressed by Sellars (1953), Brandom (1994), and Gupta (1999).

${ }^{2}$ The same point applies to Kitcher's (1982) earlier account of the gene concept. Kitcher's case study mentions three modes of reference, but he does not offer an argument for why he views them as modes of reference. As far as the general semantic account is concerned, Kitcher stresses that a speaker's intention are important for determining which mode of reference obtains: he assumes that a description is reference fixing iff a speaker intends it to be reference fixing. However, I feel that this account is in need of elaboration.
} 
${ }^{3}$ Research in genetics and genomics during the last two decades revealed unforeseen complexities, in particular the fact that genes are a very heterogeneous kind. As a consequence, a sort of fragmentation of the molecular gene concept took place, and some have argued that there are distinct gene concepts used in molecular biology. This conceptual variation in the gene concept has been extensively debated in the last few years and is still an important topic for philosophical investigation (Moss 2002; Beurton et al. 2003; Stotz and Griffiths 2004). Unfortunately, Weber does not address this issue.

${ }^{4}$ The notion of material inference as described in the previous section fits with this latter idea: a person makes a certain inference based on the content of the premisses and the conclusions involved, which depends on the content of the concepts involved. Accounts of concepts in cognitive science, in particular the psychology of concepts, also underwrite the idea that concepts figure in inference and explanation.

${ }^{5}$ This chapter includes a discussion of laws, natural kinds, and functional explanation. One of Weber's points is to show that while molecular biologists give funtional explanations, functional explanation is compatible with Weber's reductionist, law-based account of explanation - functional explanations simply makes a different use of laws. In what follows, I will not discuss this issue.

${ }^{6}$ Among others, researchers working in the field of evolutionary developmental biology view the explanation of development in this way, as due to their evolutionary focus they have to study the development of larger groups of organisms, such as the development of organism sharing a body plan.

${ }^{7}$ For a more detailed defense of non-reductionism in developmental biology, see Laubichler and Wagner (2001), Moss (2002), and Robert (2004).

\section{Acknowledgements}

I thank Alan Love and Kim Sterelny for helpful comments on a draft of this essay.

\section{References}

Beurton, P. J., R. Falk, and H.-J. Rheinberger (eds.): 2000, The Concept of the Gene in Development and Evolution. Cambridge: Cambridge University Press.

Boyd, R.: 1991, 'Realism, Anti-Foundationalism and the Enthusiasm for Natural Kinds'. Philosophical Studies 61, 127-148. 
Brandom, R. B.: 1994, Making It Explicit: Reasoning, Representing, and Discursive Commitment. Cambridge, MA: Harvard University Press.

Darden, L.: 1991, Theory Change in Science: Strategies from Mendelian Genetics. New York: Oxford University Press.

Delehanty, M.: forthcoming, 'Emergent Properties and the Context Objection to Reduction'. Biology and Philosophy.

Fodor, J.: 1975, The Language of Thought. New York: Crowell.

Frost-Arnold, G.: 2004, 'How to Be an Anti-Reductionist about Developmental Biology: Response to Laubichler and Wagner'. Biology and Philosophy 19, 75-91.

Goosens, W. K.: 1978, 'Reduction by Molecular Genetics'. Philosophy of Science 45, 73-95.

Grasshof, G. and M. May: 1995, 'Methodische Analyse wissenschaftlichen Entdeckens'. Kognitionswissenschaft 5, 51-67.

Gupta, A.: 1999, 'Meaning and Misconceptions'. In: R. Jackendoff, P. Bloom, and K. Wynn (eds.): Language, Logic, and Concepts. Cambridge, MA: MIT Press, pp. 15-41.

Holmes, F. L.: 1991, Hans Krebs. Volume I: The Formation of a Scientific Life, 1900-1933. Oxford: Oxford University Press.

Holmes, F. L.: 2000, 'The Logic of Discovery in the Experimental Life Sciences'. In: R. Creath and J. Maienschein (eds.): Biology and Epistemology. Cambridge: Cambridge University Press, pp. $167-190$.

Hull, D.: 1974, Philosophy of Biological Science. Englewood Cliffs: Prentice-Hall.

Kimbrough, S. O.: 1978, 'On the Reduction of Genetics to Molecular Biology'. Philosophy of Science 46, 389-406.

Kitcher, P.: 1978, 'Theories, Theorists, and Theoretical Change'. The Philosophical Review 87, 519547.

Kitcher, P.: 1982, 'Genes'. British Journal for the Philosophy of Science 33, 337-359.

Kitcher, P.: 1984, '1953 and All That: A Tale of Two Sciences'. The Philosophical Review 93, 335373.

Laubichler, M. and G. P. Wagner: 2001, 'How Molecular is Molecular Developmental Biology? A Reply to Alex Rosenberg's Reductionism Redux: Computing the Embryo'. Biology and Philosophy 16, 53-68.

Machamer, P., L. Darden, and C. Craver: 2000, 'Thinking About Mechanisms'. Philosophy of Science $67,1-25$.

Mayo, D. G.: 1996, Error and the Growth of Experimental Knowledge. Chicago: University of Chicago Press.

Moss, L.: 2002, What Genes Can't Do. Cambridge, MA: MIT Press.

Norton, J. D.: 2003, ‘A Material Theory of Induction'. Philosophy of Science 70, 647-670. 
Robert, J.: 2004, Embryology, Epigenesis, and Evolution: Taking Development Seriously. Cambridge: Cambridge University Press.

Ruse, M.: 1973, The Philosophy of Biology. London: Hutchinson University Library.

Schaffner, K. F.: 1974, 'Logic of Discovery and Justification in Regulatory Genetics'. Studies in History and Philosophy of Science 4, 349-385.

Schaffner, K. F.: 1993, Discovery and Explanation in Biology and Medicine. Chicago: University of Chicago Press.

Sellars, W.: 1953, 'Inference and Meaning'. Mind 1962, 313-338.

Stotz, K. and P. E. Griffiths: 2004, 'Genes: Philosophical Analyses Put to the Test'. History and Philosophy of the Life Sciences 26: 5-28. 